\title{
Efeito agudo de exercícios de alongamento estático e dinâmico na impulsão vertical de jogadores de futebol
}

\author{
Vinicius de Souza Ferreira ${ }^{1}$ \\ Bruno Colombo Muller ${ }^{2}$ \\ Abdallah Achour Junior ${ }^{3}$ \\ ${ }^{1}$ Rede Euroamericana de Motricidade Humana, Rio de Janeiro, RJ, Brasil \\ ${ }^{2}$ Curso de Educação Física, Faculdade Cenecista de Osório, RS, Brasil \\ ${ }^{3}$ Centro de Educação Física e Esporte, Departamento de Ciências do Esporte, \\ Universidade Estadual de Londrina, PR, Brasil
}

\begin{abstract}
Resumo: O objetivo deste estudo foi comparar o efeito agudo dos exercícios de alongamento estático e do alongamento dinâmico na impulsão vertical e amplitude do movimento de 22 jogadores de futebol profissional do sexo masculino. Observou-se que após a intervenção do alongamento dinâmico, o grupo experimental $(n=13)$ apresentou um aumento significativo na impulsão vertical $(p=0,002)$, enquanto que após o alongamento estático, não houve alteração significativa $(p=0,343)$, assim como na condição sem alongamento $(p>0,05)$. Com relação aos níveis de amplitude de movimento $(n=13)$, não houve diferenças significativas no sentar e alcançar $(p=0,263)$ nas três condições analisadas: alongamento estático, dinâmico e controle. Os resultados sugerem que o alongamento dinâmico é o mais indicado para ser realizado antes de atividades que exijam potência muscular, entretanto as intervenções propostas neste estudo não evidenciaram aumento na amplitude de movimento.
\end{abstract}

Palavras-chave: Amplitude de movimento. Potência muscular. Futebol.

Acute effects of static versus dynamic stretching on the vertical jump performance of soccer players

\begin{abstract}
This study aimed to compare the acute effects of static and dynamic stretching exercises on the vertical jump performance and range of motion of 22 male professional soccer players. After the dynamic stretching intervention, the experimental group $(n=13)$ showed a significant increase in vertical jump performance $(p=0,002)$, but no significant changes were observed after static stretching $(p=0,343)$ as well as after tests without stretching intervention $(p>0,05)$. Regarding range of motion $(n=13)$, there were no significant differences in the sit and reach $(p=0,263)$ under the three conditions examined: static stretching, dynamic stretching, and control (no stretching). These results suggest that dynamic stretching is the most suitable type of stretching to perform before warm-up routines that require muscle power. However, none of the interventions proposed in this study resulted in an increase in range of motion.
\end{abstract}

Keywords: Range of motion. Muscle power. Soccer.

\section{Introdução}

Os exercícios de alongamento compõem o processo de aquecimento de esportistas

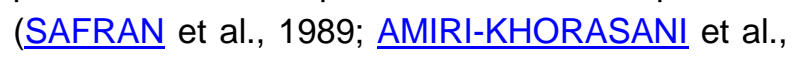
2010; GELEN, 2010).

No entanto, na última década, uma série de estudos questionou os benefícios dos exercícios do alongamento, reportando prejuízos na produção de força em diferentes populações (MAREK et al., 2005; BRADLEY et al., 2007; BRANDENBURG et al., 2007; SAYERS et al., 2008; GREGO NETO; MANFFRA, 2009) e sugerindo que não deveriam ser realizados em rotinas de aquecimento, antes de atividades que exigissem força e potência muscular (BRADLEY et al., 2007; SAYERS et al., 2008; ENDLICH et al., 2009).
Alguns autores atribuíram os resultados negativos reportados em seus estudos à intensidade máxima dos movimentos (FOWLES et al., 2000; CRAMER et al., 2005), enquanto outros, ao tempo de execução dos exercícios (SIATRAS et al., 2008; GREGO NETO; MANFFRA, 2009).

Entretanto, os exercícios de alongamento realizados com tempos elevados e intensidade máxima não refletem a rotina de aquecimento de esportistas (RODRÍGUEZ; ANDÚJAR, 2010). Estudos em que se entrevistaram treinadores de elite das principais ligas de esportes coletivos norte-americanas reportaram que, durante 0 aquecimento, cada exercício de alongamento realizado por atletas de basquete antes de uma partida dura em média 14,5 segundos (SIMENZ et 
al., 2005). Treinadores de beisebol relataram que seus jogadores realizam séries de exercícios de alongamento de em média 17 segundos (EBBEN et al., 2004). Para jogadores de hockey, a média de exercícios de alongamento encontrada foi de 12 segundos (EBBEN et al., 2005), todos em intensidade submáxima.

O alongamento estático é considerado eficiente para produzir aumento agudo na amplitude de movimento (MURPHY et al., 2010; RODRÍGUEZ; ANDÚJAR, 2010). Porém, há investigações apontando decréscimo no rendimento da força e da potência muscular (GREGO NETO; MANFFRA, 2009; AMIRIKHORASANI et al., 2010; BABAULT et al., 2010; RODRÍGUEZ; ANDÚJAR, 2010). Quando realizados em intensidade submáxima e por pouco tempo, podem não comprometer 0 desempenho da força e da potência (SIATRAS et al., 2008; WINKE et al., 2010; CANNAVAN et al., 2012).

Por outro lado, estudos recentes que compararam o efeito agudo dos exercícios de alongamento estático e dinâmico têm demonstrado que a técnica de alongamento dinâmico produz melhores resultados sobre a força e a potência muscular (AMIRI-KHORASANI et al., 2010; RODRÍGUEZ; ANDÚJAR, 2010; ALIKHAJEH et al., 2011).

No entanto, há carência de estudos que investiguem 0 efeito agudo do tempo de alongamento frequentemente usado por jogadores de futebol. Nenhum dos trabalhos citados investigou os exercícios de alongamento realizados em séries de 10 segundos, tempo comumente utilizado por esses atletas.

Tendo em vista que o futebol é um esporte que depende de potência muscular em ações multiarticulares de alta intensidade, como em sprints, saltos e agilidade (LITTLE, 2006; DELLAL et al., 2010), fica clara a necessidade de um estudo que analise as técnicas de alongamento com tempo e intensidade correspondentes às realizadas nas rotinas de aquecimento por jogadores de futebol, comparando o efeito produzido entre as séries de 10 segundos de alongamento estático e alongamento dinâmico na potência (impulsão vertical) e na amplitude de movimento.

Sendo assim, este estudo tem como propósito: a) comparar o efeito agudo dos exercícios de alongamento estático e alongamento dinâmico, realizados em séries de 10 segundos, na impulsão vertical de jogadores de futebol; b) comparar o efeito agudo dos exercícios de alongamento estático e alongamento dinâmico, realizados em séries de 10 segundos, na amplitude de movimento de jogadores de futebol.

\section{Materiais e métodos}

A presente pesquisa, de caráter descritivo comparativo, foi composta por 22 atletas de futebol profissional, do sexo masculino (média e desvio padrão; idade $=26,3 \pm 3,9$ anos; estatura $=$ $176,9 \pm 6,7$ centímetros; massa corporal = $75,7 \pm 8,1 \mathrm{~kg}$; índice de massa corporal $=24,1 \pm 1,8$ ) e integrantes de um clube participante da primeira divisão do campeonato estadual do Rio Grande do Sul, Brasil, filiado à Confederação Brasileira de Futebol. A amostra foi selecionada de forma intencional e por conveniência. Todos os participantes assinaram 0 termo de consentimento livre e esclarecido, e o clube assinou o termo de informação à instituição, como recomendado pela resolução 196/96 do Conselho Nacional de Saúde. Este estudo foi aprovado pelo Comitê de Ética da Rede Euroamericana de Motricidade Humana, sob o número 005/2011. Todos os atletas responderam negativamente aos itens do questionário de prontidão para a prática de atividade física (PAR-Q).

Foram excluídos do estudo, aqueles que apresentaram lesões ao longo dos dias em que se realizaram a coleta de dados.

\section{Equipamentos}

Em um período prévio às avaliações, foram aferidas a massa corporal dos indivíduos, em uma balança antropométrica da marca Filizola, e a estatura, em um estadiômetro marca Sanny.

Para avaliar o efeito agudo dos exercícios de alongamento estático e alongamento dinâmico na impulsão vertical, foi realizado o teste de salto vertical com contramovimento dos membros inferiores e sem a ajuda dos braços (counter movement jump), utilizando-se o tapete de contato denominado Plataforma JumpTest e conectado ao software modelo Multisprint da marca Hidrofit.

Para avaliar o efeito agudo dos exercícios de alongamento estático e alongamento dinâmico sobre a amplitude de movimento, cada indivíduo foi submetido ao teste de sentar e alcançar (WELLS; DILLON, 1952), utilizando-se o banco de Wells da marca Sanny. 


\section{Aquecimento}

Com o objetivo de reproduzir a sua realidade, os indivíduos foram submetidos a uma corrida contínua, em velocidade comumente executada em suas rotinas de aquecimento, durante 10 minutos (COLEDAM et al., 2009; RODRÍGUEZ; ANDÚJAR, 2010). Foi determinado o tempo de 10 minutos porque, segundo Stewart (1998), a temperatura muscular eleva-se rapidamente após 5 minutos, tornando-se estável após 10 minutos de exercícios contínuos.

\section{Procedimentos experimentais}

As avaliações foram realizadas em 3 dias, durante o período competitivo, e, para que não houvesse interferência de treinos e jogos, esses procedimentos foram realizados sempre no mesmo dia da semana e no horário da manhã, sem nenhum esforço prévio, com intervalo de 1 semana, após o dia de folga da equipe.

As avaliações foram feitas por quatro avaliadores experientes, graduados em Educação Física. Nas semanas anteriores, os indivíduos foram orientados e familiarizados com o protocolo de avaliação da amplitude de movimento. A avaliação da impulsão vertical fazia parte da rotina de testes dos atletas.

A amostra foi dividida de forma aleatória; 13 atletas compuseram o grupo experimental, e o grupo controle contou com 9 atletas.

O efeito agudo dos exercícios de alongamento estático e alongamento dinâmico sobre a impulsão vertical, foi avaliado pelo grupo experimental $(n=13)$ em 2 situações: sob a intervenção do alongamento estático e sob a intervenção do alongamento dinâmico no segundo e terceiro dias e pelo grupo controle nos mesmos dias $(n=9)$.

Enquanto que o efeito agudo dos exercícios de alongamento estático e alongamento dinâmico sobre a amplitude de movimento, foi avaliado pelo grupo experimental $(n=13)$ em 3 situações: sob a intervenção do alongamento estático, sob a intervenção do alongamento dinâmico e sem a intervenção dos alongamentos investigados. Conforme detalhado a seguir.

No primeiro dia de coleta, os 13 atletas do grupo experimental foram submetidos a uma avaliação para determinar a amplitude do movimento sem a intervenção dos alongamentos.
No segundo e no terceiro dia, os 13 atletas do grupo experimental foram submetidos aos testes com a intervenção do alongamento estático e do alongamento dinâmico. No entanto, para evitar que possíveis interferências decorrentes de dias distintos de coleta pudessem comprometer os resultados, o grupo experimental foi dividido de forma aleatória em dois subgrupos (grupo experimental 1 , com seis componentes, e grupo experimental 2, com sete componentes), que realizaram os alongamentos estático e dinâmico no segundo e no terceiro dia de forma inversa, conforme detalhado a seguir.

No segundo dia de coleta, o grupo experimental 1 realizou os testes de impulsão vertical antes e após a intervenção dos exercícios de alongamento estático, enquanto o grupo experimental 2 realizou os testes de impulsão vertical antes e após a intervenção dos exercícios de alongamento dinâmico, sendo que ambos os grupos foram submetidos ao sentar e alcançar após a segunda medida da impulsão vertical, realizada posteriormente aos exercícios de alongamento. $\mathrm{O}$ grupo controle realizou os testes de impulsão vertical e da amplitude do movimento sem a intervenção de alongamento.

No terceiro dia de coleta, o grupo experimental 1 realizou os testes de impulsão vertical antes e após a intervenção dos exercícios de alongamento dinâmico, enquanto 0 grupo experimental 2 realizou os testes de impulsão vertical antes e após a intervenção dos exercícios de alongamento estático, sendo que ambos os grupos foram submetidos ao sentar e alcançar após a segunda medida da impulsão vertical realizada posteriormente à intervenção dos alongamentos. O grupo controle novamente realizou os testes de impulsão vertical e amplitude do movimento sem a intervenção de alongamento.

\section{Exercícios de alongamento}

Os exercícios de alongamento selecionados para este estudo faziam parte da rotina de aquecimento dos atletas antes de treinamentos e jogos. O volume, a intensidade e a ordem de execução dos exercícios de alongamento foram os mesmos, tanto na intervenção do alongamento dinâmico como no alongamento estático, diferindo apenas a técnica de execução que caracteriza cada método. 
Todos os exercícios foram realizados em 1 série de 10 segundos de duração $\left(\begin{array}{lll}1 & x & 10\end{array}\right.$ segundos) para 0 alongamento estático, e em 1 série de 10 repetições $(1 \times 10$ repetições $)$ para o alongamento dinâmico, com intensidade submáxima, até o ponto de desconforto muscular, sendo alongado primeiramente o membro direito e após foi alongado o esquerdo em todas as ações. No alongamento estático os membros direito e esquerdo foram alternados ao término de cada série de 10 segundos, enquanto que no alongamento dinâmico os membros foram alternados a cada repetição, até totalizar 10 repetições para cada membro.

A ordem de execução dos exercícios foi a seguinte: flexão dorsal do tornozelo, flexão do quadril, extensão do quadril e flexão do joelho. Conforme ilustra a figura 1.

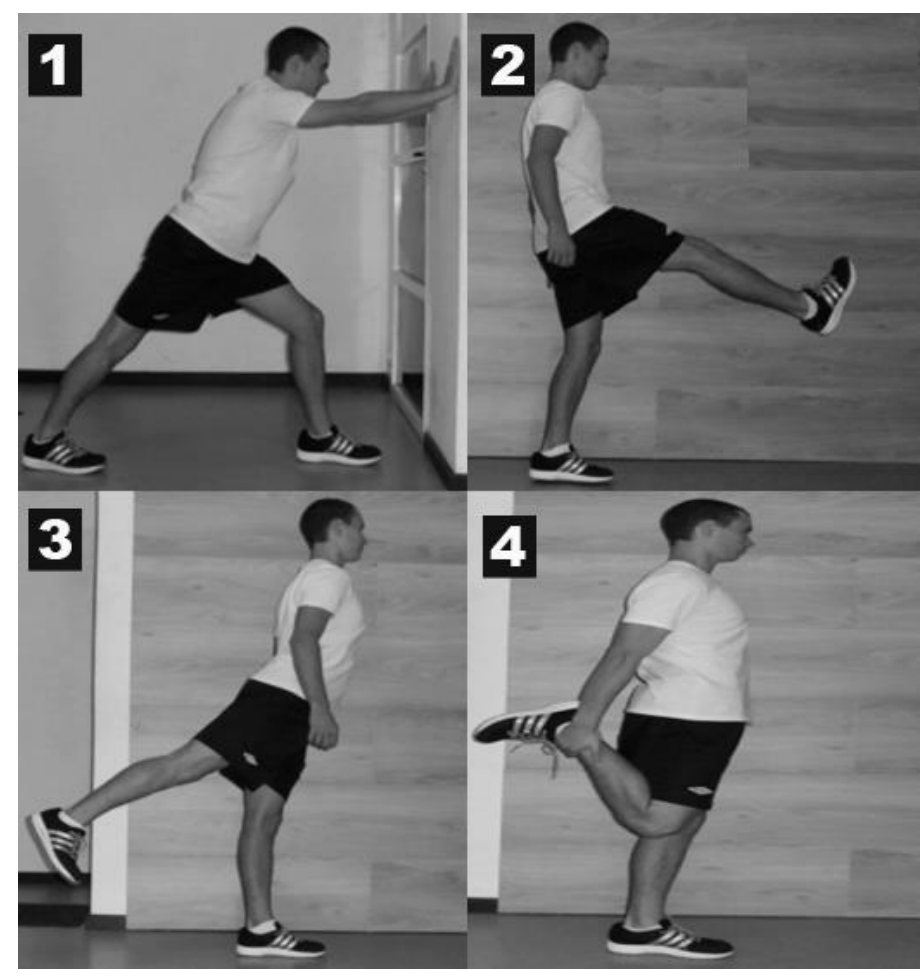

Figura1. Flexão dorsal do tornozelo (foto 1); flexão do quadril (foto 2); extensão do quadril (foto 3); flexão do joelho (foto 4).

\section{Protocolo para avaliação da impulsão vertical}

Em cada sessão, foi solicitado aos avaliados que realizassem três saltos com intervalos de 10 segundos, sendo registrada a maior medida. Para isso, o software Multisprint leva em consideração o tempo de vôo do indivíduo, que é considerado como o intervalo de tempo entre a perda de contato com o tapete e o retorno ao solo (FERREIRA et al., 2008; COELHO et al., 2011). Após mensurar o tempo de vôo, a altura do salto é calculada com base na fórmula: $h=g \cdot t^{2} \cdot 8^{-1}$ onde $\mathrm{h}$ é a altura, g é o valor da aceleração da gravidade, e t é o tempo de vôo (FERREIRA et al., 2008).

O grupo experimental realizou a avaliação imediatamente ao término do aquecimento e novamente logo após os exercícios de alongamento, enquanto o grupo controle realizou os testes sem a interferência dos exercícios de alongamento, imediatamente após o termino do aquecimento e novamente 90 segundos após a primeira coleta. Esse período reproduziu o tempo aproximado em que cada indivíduo do grupo experimental levou para realizar a intervenção dos exercícios de alongamento.

\section{Protocolo para a avaliação da amplitude de movimento}

Os atletas foram orientados a sentar no solo com os joelhos estendidos e apoiar os pés descalços na borda anterior do banco. Em seguida, foi solicitado que executassem uma flexão máxima do tronco à frente, com os cotovelos estendidos, deslizando os dedos lentamente sobre a régua fixada na parte superior do banco, até onde fosse possível (RIBEIRO et al., 2010). Foram realizadas três tentativas, sendo considerada a maior distância alcançada.

Diferentemente do teste de impulsão vertical, que foi realizado antes e após a intervenção dos exercícios de alongamento, o teste de amplitude 
de movimento foi realizado somente ao término de cada coleta. Esse critério foi adotado para evitar que a mesma pudesse influenciar no desempenho da impulsão vertical.

Dessa forma, o grupo controle e o grupo experimental realizaram o teste de amplitude de movimento no primeiro dia, na seguinte ordem: aquecimento, teste de impulsão vertical, pausa de 90 segundos sem a intervenção dos exercícios de alongamento e novamente teste de impulsão vertical. No segundo e no terceiro dia, a amplitude de movimento do grupo experimental foi testada após os procedimentos de aquecimento, impulsão vertical, intervenção dos exercícios de alongamento propostos e novamente impulsão vertical, enquanto o grupo controle realizou o teste de amplitude de movimento posteriormente aos protocolos de aquecimento, impulsão vertical, pausa de 90 segundos (tempo médio para a realização dos exercícios de alongamento investigados) e novamente impulsão vertical.

\section{Análise estatística}

Foi aplicado o teste de normalidade de Shapiro-Wilk para verificar a normalidade dos dados. A análise dos resultados da impulsão vertical realizada pelo grupo experimental, nas condições pré e pós-alongamento estático e pré e pós-alongamento dinâmico, e pelo grupo controle, foi feita através do teste $t$ de Student para amostras pareadas. E a comparação entre os métodos de alongamento (análise inter grupos), foi feita através da ANOVA two way.
Para comparar a amplitude do movimento nos exercícios de alongamento estático, alongamento dinâmico e sem alongamento, foi utilizada uma ANOVA one way, possibilitando assim, tratar a correlação existente entre as medidas repetidas de um mesmo indivíduo. Para as comparações múltiplas foi utilizado o teste de Tukey.

Os resultados foram obtidos através do software SPSS versão 20. Adotou-se o valor de $p<0,05$ para significância estatística.

\section{Resultados}

\section{Impulsão Vertical}

A Tabela 1 mostra a análise da impulsão vertical realizada no segundo e no terceiro dia de coleta pelo grupo experimental, nas condições pré e pós-alongamento estático e pré e pósalongamento dinâmico, e pelo grupo controle. Através do teste $t$ de Student verificou-se que o grupo experimental, após a intervenção do alongamento estático, não apresentou diferença estatisticamente significativa (pré-alongamento estático $39,2 \pm 4,4 \times$ pós-alongamento estático $39,6 \pm 4,2$ centímetros, $p=0,343$ ), enquanto que, após realizar o alongamento dinâmico, o grupo experimental apresentou um aumento significativo na impulsão vertical (pré-alongamento dinâmico $39,8 \pm 4,6 \times$ pós-alongamento dinâmico $41,5 \pm 4,3$ centímetros, $\mathrm{p}=0,002$ ).

Tabela 1. Resultados comparativos da impulsão vertical $(\mathrm{cm})$ do grupo experimental, nas condições pré e pós-alongamento estático e pré e pós- alongamento dinâmico, e pelo grupo controle.

\begin{tabular}{|l|l|l|l|l|}
\hline \multicolumn{1}{|c|}{ Grupos } & Tempo & $\mathbf{N}$ & Média & D.P \\
\hline \multirow{2}{*}{ Alongamento Dinâmico } & Pré & 13 & 39,85 & 4,62 \\
\cline { 2 - 5 } & Pós & 13 & 41,59 & $4,35^{*}$ \\
\hline \multirow{2}{*}{ Alongamento Estático } & Pré & 13 & 39,20 & 4,28 \\
\cline { 2 - 5 } & Pós & 13 & 39,64 & 4,46 \\
\hline \multirow{2}{*}{ Grupo Controle } & Pré & 09 & 41,64 & 5,15 \\
\cline { 2 - 5 } & Pós & 09 & 41,91 & 5,54 \\
\hline
\end{tabular}

${ }^{*}$ Condição de impulsão vertical significativamente maior do que na condição pré teste $(p=0,002)$

A tabela 2 apresenta $o$ resultado da comparação entre os métodos de alongamento (análise inter grupos). Ao solicitar a ANOVA two way, verificou-se que a condição de alongamento dinâmico apresentou uma média da impulsão vertical $1,3 \mathrm{~cm}$ acima da condição alongamento estático, sendo essa diferença significativa $(p=0,005)$. Entretanto, não existem evidências significativas de que as condições de alongamento dinâmico e alongamento estático sejam diferentes da condição controle. (comparação entre alongamento dinâmico e controle $(p=0,9139)$. Comparação entre alongamento estático e controle: $(p=0,4734)$. 
Tabela 2. Resultado da comparação da impulsão vertical $(\mathrm{cm})$ entre os métodos de alongamento (análise inter grupos).

\begin{tabular}{ccccc}
\hline Comparações Múltiplas & Diferença das médias & Erro Padrão & P-valor \\
\hline & Alongamento Estático - Alongamento Dinâmico & $-1,296$ & 0,410 & 0,0052 \\
& Alongamento Dinâmico - Grupo Controle & 1,060 & 1,780 & 0,9139 \\
& Alongamento Estático - Grupo Controle & 2,356 & 1,780 & 0,4736 \\
\hline Tempo & Pós - Pré & 0,816 & 0,319 & 0,0200 \\
\hline
\end{tabular}

Tabela 3. Resultados comparativos da amplitude de movimento $(\mathrm{cm})$ nas condições: a) alongamento estático; b) alongamento dinâmico; c) sem alongamento.

\begin{tabular}{lccc}
\hline Intervenção & N & Média & D.P \\
\hline Alongamento Dinâmico & 13 & 36,48 & 4,00 \\
Alongamento Estático & 13 & 35,33 & 5,31 \\
Sem Alongamento & 13 & 34,79 & 4,19 \\
\hline
\end{tabular}

\section{Amplitude de movimento}

A tabela 3 mostra a análise da amplitude de movimento $(\mathrm{cm})$ nas condições: a) alongamento estático; b) alongamento dinâmico; c) sem alongamento. Por meio dos resultados da ANOVA one way, observou-se que não existem diferenças significantes entre os resultados observados nas três medições $(p=0,1328)$.

\section{Discussão}

O objetivo deste estudo foi comparar o efeito agudo do exercício de alongamento estático e do alongamento dinâmico, realizados em séries de 10 segundos na impulsão vertical e amplitude do movimento de jogadores de futebol profissional do sexo masculino.

Os dados das Tabelas 1 e 2 mostraram que 0 protocolo de alongamento estático não produziu efeito negativo na impulsão vertical, concordando com outros estudos que não relataram prejuízo na potência muscular, sobretudo aqueles realizados em intensidade submáxima e séries inferiores a 45 segundos (WINKE et al., 2010; CANNAVAN et al., 2012)

Estudos sugerem que o alongamento estático mostra-se mais efetivo na ativação de mecanorreceptores responsáveis por gerar relaxamento da tensão viscoelástica da unidade musculotendínea, além de maior ativação dos reflexos inibitórios gerados pelos órgãos tendinosos de Golgi (KISTLER et al., 2010; MCHUGH; COSGRAVE, 2010), o que parece ser contraproducente para a geração de força e potência muscular havendo uma relação direta com o tempo de duração destes exercícios (ZAKAS et al., 2006; BRANDENBURG et al., 2007; GREGO NETO; MANFFRA, 2009)..

Conforme demonstra o estudo de Grego Neto e Manffra (2009) que avaliaram o efeito de quatro séries de 45 segundos (tempo total de 180 segundos) e oito séries de 45 segundos (tempo total de 360 segundos) de alongamento estático. Observaram que ambos os protocolos promoveram ganho de flexibilidade em níveis semelhantes, contudo a diminuição no pico de torque e trabalho máximo foi mais significativa nos indivíduos submetidos ao protocolo com volume total de 360 segundos. Os autores sugeriram que as alterações na rigidez muscular que causaram ganho na flexibilidade, não seriam os únicos responsáveis pelos déficits de força. Concluindo também que a capacidade máxima de gerar força é dependente do volume de alongamento, mas a produção de trabalho ao longo de algumas repetições não causa efeitos deletérios.

No estudo de Zakas e colaboradores (2006), o volume de alongamento, também foi um fator determinante para o déficit no pico de torque de 14 jogadores de futebol, sendo que os mesmos passaram por três situações distintas de alongamento estático. O primeiro protocolo de alongamento foi realizado em uma única série de 30 segundos; o segundo, em 10 séries 30 segundos (tempo total de 300 segundos); e o terceiro, em 16 séries de 30 segundos (tempo total de 480 segundos). Os autores concluíram que a série única de 30 segundos não comprometeu os níveis de força em todas as 
velocidades angulares, diferentemente dos protocolos de 300 e 480 segundos que apresentaram perda de força do pico de torque em todos os ângulos.

Nosso estudo também observou que os exercícios de alongamento estático realizados em uma série de 10 segundos, em intensidade submáxima, não produziram alterações na amplitude de movimento dos indivíduos.

Com isso, acreditamos que o alongamento estático proposto em nossa pesquisa, realizado em 1 série de 10 segundos em intensidade submáxima, foi um estímulo insuficiente para produzir alterações mecânicas ou neurais capazes de gerar comprometimento na impulsão vertical e aumento na amplitude de movimento dos jogadores de futebol profissional avaliados.

Com relação aos exercícios de alongamento dinâmico, corroboramos também com os resultados de investigações que atestaram efeitos benéficos no desempenho de diferentes métodos para avaliar a força e a potência de jogadores de futebol, submetidos a este método, como na velocidade em sprints (LITTLE; WILLIAMS, 2006; GELEN, 2010; RODRÍGUEZ; ANDÚJAR, 2010; ALIKHAJEH et al., 2011), potência no chute (GELEN, 2010; AMIRI-KHORASANI et al., 2011) e agilidade (MCMILLIAN et al., 2006; AMIRIKHORASANI et al., 2010).

Estudos que compararam o efeito agudo dos exercícios de alongamento estático e dinâmico também referiram o dinâmico como sendo o mais adequado para ser realizado em rotinas de aquecimento antes de atividades esportivas que exijam potência muscular (SHELTON; KUMAR, 2009; AMIRI-KHORASANI et al., 2010; RODRÍGUEZ; ANDÚJAR, 2010; ALIKHAJEH et al., 2011; VAN GELDER; BARTZ, 2011).

No entanto, apesar do significativo número de estudos acerca do assunto, nenhum dos trabalhos supracitados avaliou o efeito agudo dos exercícios de alongamento dinâmico realizados em séries de 10 segundos. Com isso, a investigação desse protocolo mostrou-se importante. Pois, diferentemente do alongamento estático, onde os trabalhos sugerem que, quanto maior o tempo de execução, maior a possibilidade de comprometimento dos elementos periféricos e/ou neurais(MAREK et al., 2005; SIATRAS et al., 2008; GREGO NETO; MANFFRA, 2009), parece que $\mathrm{o}$ alongamento dinâmico pode promover aumento no desempenho muscular tanto em volumes maiores (MCMILLIAN et al., 2006; AMIRI-KHORASANI et al., 2011; VAN GELDER; BARTZ, 2011) quanto nas séries de 10 repetições, propostas no presente estudo, questionando assim, a necessidade da prescrição de séries de alongamento dinâmico superiores a esta, nas rotinas de aquecimento de jogadores de futebol profissional.

Acreditamos que o aumento na impulsão vertical após a intervenção do alongamento dinâmico seja decorrente do dinamismo das ações concêntricas e excêntricas executadas neste método, potencializando a excitação de receptores nervosos e consequentemente a velocidade do impulso nervoso no ciclo alongamento-encurtamento, além de possível aumento na temperatura corporal e fluxo sanguíneo nas musculaturas solicitadas.

Os dados da Tabela 3 mostram que, após os exercícios de alongamento dinâmico, não houve alterações estatisticamente significativas no sentar e alcançar. Ou seja, parece que o tempo de 10 segundos para cada série e a intensidade submáxima, proposta por este estudo, independente do método de alongamento, não foram suficientes para aumentar a amplitude de movimento, diferente de outros trabalhos que investigaram tempos superiores, como 0 de Murphy et. al., (2010), que reportaram que uma série de 20 segundos de exercícios de alongamento, tanto estático quanto dinâmico, aumentou o desempenho no sentar e alcançar em uma amostra composta por 42 homens fisicamente ativos (entretanto, as modificações nos testes de impulsão vertical não foram estatisticamente significativas: 1,7 e $2 \%$, respectivamente). Da mesma forma, Rodriguez e Andújar (2010) relataram aumento na amplitude de movimento após exercícios de alongamento estático/passivo ( $2 \times 30$ segundos), estático/ativo ( $2 \times 30$ segundos) e dinâmico ( $2 \times 15$ repetições) em jogadores de futebol.

\section{Conclusão}

Considerando os resultados encontrados, verificamos que exercícios de alongamento dinâmico realizados em séries de 10 repetições foram suficientes para promover melhora no desempenho da impulsão vertical (potência) de jogadores de futebol, não havendo a necessidade de volumes superiores de alongamento para esse fim. Entretanto, novos estudos com alongamento 
dinâmico em séries de 10 repetições devem ser realizados para uma melhor compreensão de seus efeitos sobre a amplitude de movimento.

Pois apesar de uma quantidade considerável de estudos direcionarem seus questionamentos para o efeito agudo gerado pelos exercícios de alongamento sobre a força e/ou a potência muscular, é importante salientar que, se o objetivo da prescrição das sessões de alongamento dentro de processo de aquecimento for melhorar a amplitude de movimento (paralelamente ao aumento agudo da força e/ou da potência), será preciso que mais estudos sejam realizados, para que consigamos chegar a um consenso de qual o método, volume e intensidade, são os mais indicados para serem ministrados em rotinas de aquecimento de esportistas que dependem destas duas capacidades motoras. Pois as respostas neurais e/ou mecânicas geradas nas sessões do alongamento têm sido sugeridas por diversos autores, como os fatores que geram tanto a melhora da amplitude de movimento quanto o comprometimento da força e da potencia muscular.

Sugerimos também que as próximas investigações comparem e controlem níveis de creatinaquinase, hidroxipolina, temperatura corporal e a frequência cardíaca em diferentes tempos de alongamento dinâmico para que tenhamos um melhor entendimento dos efeitos fisiológicos produzidos por essa técnica.

\section{Referências}

ALIKHAJEH, Y. et al. The effect of different warmup protocols on young soccer players' sprint.

Procedia Soc Behav Sci, Procedia, v. 30, p. 1588-1592, dec. 2011. Disponível em:

<http://www.sciencedirect.com/science/article/pii/S 1877042811021331>. Acesso em: 10 mai 2012.

AMIRI-KHORASANI, M. et al. Acute effect of static and dynamic stretching on hip dynamic range of motion during instep kicking in professional soccer players. J Strength Cond Res, Connecticut, v. 25, n. 6, p. 1647-1652, Jun. 2011. Disponível em:<

http://journals.Iww.com/nscajscr/Abstract/2011/06 000/Acute Effect of Static and Dynamic Stretc hing on.23.aspx>. Acesso em: 10 mai 2012.

AMIRI-KHORASANI, M. et al. Acute effect of different stretching methods on Illinois agility test in soccer players. J Strength Cond Res, Connecticut, v. 24, n. 10, p. 2698-2704, Oct. 2010. Disponível em:

$<$ http://journals.Iww.com/nscajscr/Abstract/2010/1
0000/Acute Effect of Different Stretching Metho ds on.17.aspx>. Acesso em: 10 mai 2012.

BABAULT, N. et al. Acute effects of $15 \mathrm{~min}$ static or contract-relax stretching modalities on plantar flexors neuromuscular properties. J Sci Med Sport, Auckland, v. 13, n. 2, p. 247-252, Mar. 2010. Disponível em:

$<$ http://issuu.com/cepdijon/docs/babault et al 20 10 isams >. Acesso em: 10 mai 2012.

BRADLEY, P. S. et al. The effect of static, ballistic, and proprioceptive neuromuscular facilitation stretching on vertical jump performance. $\mathbf{J}$ Strength Cond Res, Connecticut, v. 21, n. 1, p. 223-226, Feb. 2007. Disponível em: $<$ http://www.ncbi.nlm.nih.gov/pubmed/17313299 >. Acesso em: 20 mai 2012.

BRANDENBURG, J. et al. Time course of changes in vertical-jumping ability after static stretching. Int J Sports Physiol Perform, La Crosse, v. 2, n. 2, p. 170-181, Jun. 2007. Disponível em:

$<$ http://journals. humankinetics.com/ijsppbackissue s/lJSPPVolume2Issue2June/TimeCourseofChang esinVerticalJumpingAbilityAfterStaticStretching $>$. Acesso em: 20 mai 2012.

CANNAVAN, D. et al. Lack of effect of moderateduration static stretching on plantar flexor force production and series compliance. Clin Biomech (Bristol, Avon), Bristol, v. 27, n. 3, p. 306-312, Mar. 2012. Disponível em:

$<$ http://www.clinbiomech.com/article/S0268$0033(11) 00261-0 /$ abstract>. Acesso em: 20 mai 2012.

COELHO, D. B. et al. Correlação entre o desempenho de jogadores de futebol no teste de sprint de $30 \mathrm{~m}$ e no teste de salto vertical. Motriz, Rio Claro, v. 17, n. 1, p. 63-70, Mar. 2011. Disponível em:

$<$ http://www.periodicos.rc.biblioteca.unesp.br/inde x.php/motriz/article/view/19806574.2011v17n1p63>. Acesso em: 10 mai 2012.

COLEDAM, D. H. C. et al. Efeito do aquecimento com corrida sobre a agilidade e a impulsão vertical em jogadores juvenis de futebol. Motriz, Rio Claro, v. 15, n. 2, p. 257-262, Jun. 2009. Disponível em: $<$ http://www.periodicos.rc.biblioteca.unesp.br/inde x.php/motriz/article/view/2053>. Acesso em: 10 mai 2012.

CRAMER, J. T. et al. The acute effects of static stretching on peak torque, mean power output, electromyography, and mechanomyography. Eur J Appl Physiol, Berlim, v. 93, n. 5-6, p. 530-539, Mar. 2005. Disponível em:

$<$ http://www.springerlink.com/content/xald6t74ftye 0y2i/>. Acesso em: 20 mai 2012. 
DELLAL, A. et al. Physiologic effects of directional changes in intermittent exercise in soccer players. J Strength Cond Res, Connecticut, v. 24, n. 12, p. 3219-3226, Dec. 2010. Disponível em: <http://www.ncbi.nlm.nih.gov/pubmed/19996785>. Acesso em: 20 mai 2012.

EBBEN, W. P. et al. Strength and conditioning practices of National Hockey League strength and conditioning coaches. J Strength Cond Res, Connecticut, v. 18, n. 4, p. 889-897, Nov. 2004. Disponível em:

<http://www.ncbi.nlm.nih.gov/pubmed/15574099>. Acesso em: 20 mai 2012.

EBBEN, W. P. et al. Strength and conditioning practices of Major League Baseball strength and conditioning coaches. J Strength Cond Res, Connecticut, v. 19, n. 3, p. 538-546, Aug. 2005. Disponível em:

<http://www.ncbi.nlm.nih.gov/pubmed/16095401>. Acesso em: 20 mai 2012.

ENDLICH, P. W. et al. Acute effects of static stretching in dynamic force performance in young men. Rev Bras Med Esporte, São Paulo, v. 15, n. 3, p. 200-203, 2009. Disponível em:

$<$ http://www.scielo.br/scielo.php?script=sci abstra ct\&pid=S1517-

$86922009000300007 \& \operatorname{lng}=\mathrm{pt} \& \mathrm{nrm}=\mathrm{iso} \& \operatorname{lng}=\mathrm{pt}>$. Acesso em: 20 mai 2012.

FERREIRA, J. C. et al. Validade e confiabilidade de um tapete de contato para mensuração da altura do salto vertical. Braz J Biomech, São Paulo, v. 9, n. 17, 2008. Disponível em: $<$ http://citrus.uspnet.usp.br/biomecan/ojs/index.ph p/rbb/article/view/74>. Acesso em: 20 mai 2012.

FOWLES, J. R. et al. Reduced strength after passive stretch of the human plantarflexors. $\mathbf{J}$ Appl Physiol, Bethesda, v. 89, n. 3, p. 11791188, Sep. 2000. Disponível em: $<$ http://jap.physiology.org/content/89/3/1179.full>. Acesso em: 20 mai 2012.

GELEN, E. Acute effects of different warm-up methods on sprint, slalom dribbling, and penalty kick performance in soccer players. J Strength Cond Res, Connecticut, v. 24, n. 4, p. 950-956, Apr. 2010. Disponível em: $<$ http://journals.Iww.com/nscajscr/Abstract/2010/0 $4000 /$ Acute Effects of Different Warm Up Meth ods on.9.aspx>. Acesso em: 20 mai 2012.

GREGO NETO, A.; MANFFRA, E. F. Influence of static stretching volume in isokinetic variables of harmstrings. Rev Bras Med Esporte, São Paulo, 15, n. 2, p. 104-109, 2009. Disponível em: $<$ http://www.scielo.br/scielo.php?script=sci abstra ct\&pid=S1517-

$86922009000200004 \&$ lng $=p t \& n r m=i s o \& t \mid n g=p t>$. Acesso em: 20 mai 2012.
KISTLER, B. M. et al. The acute effects of static stretching on the sprint performance of collegiate men in the 60- and 100-m dash after a dynamic warm-up. J Strength Cond Res, Connecticut, v. 24, n. 9, p. 2280-2284, Sep. 2010. Disponível em: $<$ http://journals.Iww.com/nscajscr/Abstract/2010/0 9000/The Acute Effects of Static Stretching on the.3.aspx>. Acesso em: 20 mai 2012.

LITTLE, T.; WILLIAMS, A. G. Effects of differential stretching protocols during warm-ups on highspeed motor capacities in professional soccer players. J Strength Cond Res, Connecticut, v. 20, n. 1, p. 203-207, Feb. 2006. Disponível em: <http://journals.Iww.com/nscajscr/Abstract/2006/0 2000/Effects of Differential Stretching Protocols. 33.aspx>. Acesso em: 20 mai 2012.

MAREK, S. M. et al. Acute Effects of Static and Proprioceptive Neuromuscular Facilitation Stretching on Muscle Strength and Power Output. J Athl Train, Dallas, v. 40, n. 2, p. 94-103, Jun. 2005. Disponível em:

$<$ http://www.ncbi.nlm.nih.gov/pubmed/15970955>. Acesso: 20 mai 2012.

MCHUGH, M. P.; COSGRAVE, C. H. To stretch or not to stretch: the role of stretching in injury prevention and performance. Scand J Med Sci Sports, Copenhagen, v. 20, n. 2, p. 169-181, Apr. 2010. Disponível em:

$<$ http://onlinelibrary.wiley.com/doi/10.1111/j.16000838.2009.01058.x/full>. Acesso em: 20 mai 2012.

MCMILLIAN, D. J. et al. Dynamic vs. staticstretching warm up: the effect on power and agility performance. J Strength Cond Res, Connecticut, v. 20 , n. 3, p. 492-9, Aug. 2006. Disponível em: $<$ http://journals.Iww.com/nscajscr/Abstract/2006/0 8000/Dynamic Vs Static Stretching Warm Up the Effect.6.aspx>. Acesso em: 20 mai 2012.

MURPHY, J. C. et al. Effect of single set dynamic and static stretching exercise on jump height in college age recreational athletes. Int $\mathbf{J}$ Exerc Sci, Lexington, v. 3, n. 4, p. 215-224, 2010. Disponível em:

$<$ http://digitalcommons.wku.edu/ijes/vol3/iss4/8/>. Acesso em: 10 mai 2012.

RIBEIRO, C. C. A. et al. Level of flexibility through sit and reach test from research performed in São Paulo city Rev Bras Cineantropom

Desempenho Hum, Florianópolis, v. 12, n. 6, p. 415-421, 2010. Disponível em:

$<$ http://www.rbcdh.ufsc.br/MostraEdicao.do?edica o=50>. Acesso em: 20 mai 2012.

RODRíGUEZ, F. A.; ANDÚJAR, P. S. B. Efecto agudo del estiramiento sobre el sprint en jugadores de fútbol de división de honor juvenil. Revista Internacional de Ciencias del Deporte, 
Madrid, v. 18, n. 6, p. 1-12, 2010. Disponível em: <http://www.cafyd.com/REVISTA/ojs/index.php/ric yde/article/view/258>. Acesso em: 20 mai 2012.

SAFRAN, M. R. et al. Warm-up and muscular injury prevention. An update. Sports Med, Auckland, v. 8, n. 4, p. 239-249, Oct. 1989. Disponível em: $<$ http://ukpmc.ac.uk/abstract/MED/2692118/reload $=0$;jsessionid $=$ pOKozvXj50f9JXIFzBwk.2>. Acesso em: 20 mai 2012.

SAYERS, A. L. et al. The effect of static stretching on phases of sprint performance in elite soccer players. J Strength Cond Res, Connecticut, v. 22, n. 5, p. 1416-1421, Sep. 2008. Disponível em: $<$ http://journals.Iww.com/nscaiscr/Abstract/2008/09000/The Effect of Static St retching on Phases of.5.aspx>. Acesso em: 20 mai 2012.

SHELTON, J.; KUMAR, G. V. P. Comparison between static and dynamic warm-up exercise regimes on lower limb muscle power. Health, Irvine, v. 1, n. 2, p. 117-120, 2009. Disponível em: $<$ http://www.scirp.org/journal/Paperlnformation.as px?paper|D=711 >. Acesso em: 20 mai 2012.

SIATRAS, T. A. et al. The duration of the inhibitory effects with static stretching on quadriceps peak torque production. J Strength Cond Res, Connecticut, v. 22, n. 1, p. 40-46, Jan. 2008. Disponível em:

<http://journals.Iww.com/nscascr/Abstract/2008/01 $000 /$ The Duration of the Inhibitory Effects with Static.8.aspx>. Acesso em: 20 mai 2012.

SIMENZ, C. J. et al. Strength and conditioning practices of National Basketball Association strength and conditioning coaches. J Strength Cond Res, Connecticut, v. 19, n. 3, p. 495-504, Aug. 2005. Disponível em: <http://journals.Iww.com/nscajscr/Abstract/2005/0 8000/Strength and Conditioning Practices of $\mathrm{N}$ ational.3.aspx>. Acesso em: 20 mai 2012.

STEWART, I. B.; SLEIVERT, G. G. The effect of warm-up intensity on range of motion and anaerobic performance. J Orthop Sports Phys Ther, Washington, v. 27, n. 2, p. 154-161, Feb. 1998. Disponível em:

$<$ http://www.jospt.org/issues/articleID.608,type.2/a rticle detail.asp>. Acesso em: 20 mai 2012.

TAYLOR, K. L. et al. Negative effect of static stretching restored when combined with a sport specific warm-up component. J Sci Med Sport, Victoria, v. 12, n. 6, p. 657-661, Nov. 2009. Disponível em: <http://www.ncbi.nlm.nih.gov/pubmed/18768355>. Acesso em: 20 mai 2012.
VAN GELDER, L. H.; BARTZ, S. D. The effect of acute stretching on agility performance. $\mathbf{J}$

Strength Cond Res, Connecticut, v. 25, n. 11, p. 3014-3021, Nov. 2011. Disponível em:

$<$ http://journals.lww.com/nscajscr/Abstract/2011/1 $1000 /$ The Effect of Acute Stretching on Agility. 11.aspx>. Acesso em: 20 mai 2012.

WELLS, K. F.; DILLON, E. K. The sit and reach: a test of back and leg flexibility. Res Q Exerc Sport, Reston, v. 23, p. 115-118, 1952.

WINKE, M. R. et al. Moderate static stretching and torque production of the knee flexors. J Strength Cond Res, Connecticut, v. 24, n. 3, p. 706-710, Mar. 2010. Disponível em: <http://journals.Iww.com/nscajscr/Fulltext/2010/03 $000 /$ Moderate Static Stretching and Torque Pro duction.16.aspx>. Acesso em: 20 mai 2012.

ZAKAS, A. et al. Effect of two acute static stretching durations of the rectus femoris muscle on quadriceps isokinetic peak torque in professional soccer players. Isokinet Exerc Sci, Birmingham, v. 14, n. 4, p. 357-362, 2006. Disponível em: $<$ http://iospress.metapress.com/content/e5rg2mr8 m9buh1r9/?p=95c0d9d97d2c4f7cb22cd5f6455ec8 b4\&pi=7>. Acesso em: 20 mai 2012.

\author{
Endereço: \\ Vinicius de Souza Ferreira \\ Rua Pernambuco, 1556 Bairro São José \\ Tramandaí RS Brasil \\ 95590-000 \\ Telefone: (51) 3684-3401 \\ e-mail: contato@viniciusferreira.com
}

Recebido em: 23 de julho de 2012.

Aceito em: 19 de abril de 2013.

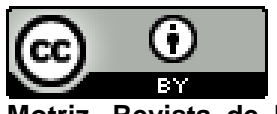

Motriz. Revista de Educação Física. UNESP, Rio Claro, SP, Brasil - elSSN: 1980-6574 - está licenciada sob Creative Commons - Atribuição 3.0 